\title{
THE DETERMINATION OF STRESSES FROM STRAINS ON THREE INTERSECTING GAGE LINES AND ITS APPLICATION TO ACTUAL TESTS
}

\author{
By Wm. R. Osgood and Rolland G. Sturm ${ }^{1}$
}

\section{ABSTRACT}

The paper presents a graphical construction for determining in problems of plane stress the state of strain at a point from measured strains taken on three gage lines intersecting at the point. The method is based on the dyadic circle, first used by Otto Mohr for determining moments of inertia, later applied by Robert Land to the plane state of stress, and finally generalized by $\mathrm{H}$. M. Westergaard.

Applications of the method to various projects of the Aluminum Research Laboratories are noted, and examples of the use of the method in determining the state of strain in the end batten plate of a column are given.

\section{CONTENTS}

I. Theory

II. Applications to actual tests

\section{THEORY}

Not infrequently in cases of plane stress it is desired to determine the state of stress at a point from strains taken on short gage lines through the point. Usually the strains in such cases have been measured on four gage lines $45^{\circ}$ apart, and the results interpreted, sometimes by cumbersome means. Although it is desirable where possible to take readings on four gage lines to obtain a check, only three gage lines are actually necessary. This obviates the necessity of preparing a fourth gage line, taking readings on it, and adjusting observations later, which in some cases may be inconvenient or impossible. The 3-gage-line method does not appear to be known widely-so far as the authors are aware, it has been disseminated primarily by Prof. H. M. Westergaard in his classes in the theory of elasticity. The only known published suggestion of the method appears in one of his papers, ${ }^{2}$ but the application specifically to strains is not given there.

In the case of plane stress the equations expressing the relation between the components of strain at a point referred to any pair of rectangular axes, $x^{\prime}$ and $y^{\prime}$, parallel to the plane of stress and the components of the same strain referred to any other pair of rectangular axes, $x$ and $y$, parallel to the piane of stress are ${ }^{3}$

$$
\epsilon^{\prime}{ }_{x}=\frac{1}{2}\left(\epsilon_{x}+\epsilon_{y}\right)+\frac{1}{2}\left(\epsilon_{x}-\epsilon_{y}\right) \cos 2 \psi+\frac{1}{2} \gamma \sin 2 \psi
$$

\footnotetext{
1 Research engineer-physicist, Aluminum Co. of America, New Kensington, $\mathrm{Pa}$.

2 Anwendung der Statik auf die Augsleichungsrechnung, Göttingen, p. 37, 1925.

3 Fuller and Johnston, Applied Mechanics, vol. 2, art. 42, John Wiley \& Sons, New York.
} $167156-33-8$ 


$$
\begin{aligned}
\epsilon^{\prime}{ }_{y} & =\frac{1}{2}\left(\epsilon_{x}+\epsilon_{y}\right)-\frac{1}{2}\left(\epsilon_{x}-\epsilon_{y}\right) \cos 2 \psi-\frac{1}{2} \gamma \sin 2 \psi \\
\gamma^{\prime} & =\left(\epsilon_{y}-\epsilon_{x}\right) \sin 2 \psi+\gamma \cos 2 \psi
\end{aligned}
$$

where

$\epsilon_{x}$ and $\epsilon_{y}$ are the components of strain in the directions $x$ and $y$, respectively

$\gamma$ is the shearing strain with respect to the axes of $x$ and $y$ $\psi$ is the angle measured positive counterclockwise from the positive axis of $x$ to the positive axis of $x^{\prime}$

$\epsilon^{\prime}{ }_{x}$ and $\epsilon^{\prime}{ }_{y}$ are the components of strain in the directions $x^{\prime}$ and $y^{\prime}$, respectively

and

$\gamma^{\prime}$ is the shearing strain with respect to the axes of $x^{\prime}$ and $y^{\prime}$.

If now we know the strains $\epsilon_{1}, \epsilon_{2}, \epsilon_{3}$ at a point in three directions making the angles $\psi_{1}, \psi_{2}, \psi_{3}$, respectively, with any axis $x$, as in figure $1(a)$, we may determine the state of strain at the point by the following construction: ${ }^{4}$

From any point $T$, as in figure $1(b)$, lay off $\epsilon_{1}, \epsilon_{2}, \epsilon_{3}$ in directions making angles $2 \psi_{1}, 2 \psi_{2}, 2 \psi_{3}$, respectively, with the negative direction of the axis of $x$. A negative strain (a shortening) should be laid off from $T$ in the opposite direction from that indicated; that is, making an angle $2 \psi \pm \pi$ with the negative direction of the axis of $x$. (The strains laid off in figure $1(b)$ are positive (elongations).) At the end points of $\epsilon_{1}, \epsilon_{2}, \epsilon_{3}$ draw the three perpendiculars $P_{1}, P_{2}, P_{3}$, respectively, and in each case shade the side away from $T$ if the corresponding strain is positive and toward $T$ if the corresponding strain is negative. Now draw the circle which is tangent to the three perpendiculars either on all the shaded sides or on all the unshaded sides. (In general, it will be possible to draw four circles tangent to the three perpendiculars, but only one will satisfy the condition which has been imposed.) Through the center of the circle draw the axis $O x$, and tangent to the circle the axis $O y$, taking $O$ so that the positive axis of $x$ intersects the circle if the latter is tangent on the unshaded sides of $P_{1}, P_{2}, P_{3}$, and so that the negative axis of $x$ intersects the circle if the latter is tangent on the shaded sides of $P_{1}, P_{2}, P_{3}$. The state of strain is determined completely by this construction. ${ }^{5}$ For, from $T$ drop the perpendicular $7 R$ on the axis of $x$. The strains $\epsilon_{x}, \epsilon_{y}$, and $\gamma$ may be determined from the following three equations, obtained by substituting $\epsilon_{1}, \psi_{1} ; \epsilon_{2}, \psi_{2} ; \epsilon_{3}, \psi_{3}$ in equation (1) three times successively

$$
\begin{aligned}
& \epsilon_{1}=\frac{1}{2}\left(\epsilon_{x}+\epsilon_{y}\right)+\frac{1}{2}\left(\epsilon_{x}-\epsilon_{y}\right) \cos 2 \psi_{1}+\frac{1}{2} \gamma \sin 2 \psi_{1} \\
& \epsilon_{2}=\frac{1}{2}\left(\epsilon_{x}+\epsilon_{y}\right)+\frac{1}{2}\left(\epsilon_{x}-\epsilon_{y}\right) \cos 2 \psi_{2}+\frac{1}{2} \gamma \sin 2 \psi_{2} \\
& \epsilon_{3}=\frac{1}{2}\left(\epsilon_{x}+\epsilon_{y}\right)+\frac{1}{2}\left(\epsilon_{x}-\epsilon_{y}\right) \cos 2 \psi_{3}+\frac{1}{2} \gamma \sin 2 \psi_{3} .
\end{aligned}
$$

' First used for determining moments of inertia by O. Mohr, Abhandlungen aus dem Gebiete der technlschen Mochanik, H ed., D. On, Wilhelm Ernst und Sohn, Berlin, 192s; later applied to the plane state of streas by Kobt land, Z.eitschrift des Vereines deutscher Ingenieure, vol. 39, p. 1551, Dec. 28, 1895. 
The values $\epsilon_{x}=O R, \epsilon_{y}=R X, \gamma=2 R T$ satisfy these three equations, as may be seen in the case of the first equation, for example, by drawing the diameter parallel to $\epsilon_{1}$ in figure $1(b)$, dropping the perpendicular $T R_{1}$ on it, and noting that $\epsilon_{1}$ is equal to the projection of $Y_{1} M R T$ on this diameter. Furthermore, the direction of $\epsilon_{1}$ appears in the circle as $O x_{1}$, which passes through $X_{1}$, the far end of the diameter parallel to $\epsilon_{1}$ - that is, the end away from $P_{1}$. The component of strain in a direction, $O y_{1}$, at right angles to that of $\epsilon_{1}$ is seen from equation (2) to be given by $R_{1} X_{1}$, and the shearing strain with respect to the axes $x_{1}, y_{1}$ is seen from equation (3) to be given by $2 R_{1} T$.

If the circle is tangent on the unshaded sides of $P_{1}, P_{2}, P_{3}$, then strains measured from the circumference toward the center of the circle are positive, and strains measured from the circumference away from the center are negative. If the circle is tangent on the shaded sides of $P_{1}, P_{2}, P_{3}$, the opposite rule holds. The signs of the shearing strains are usually not of importance. Shearing strains with respect to any axes $x$ and $y$ are taken as positive when elements normal to the axis of $x$ and on the positive side of the axis of $y$ tend to slide in the positive direction of $y$ relative to elements normal to the axis of $x$ which contain the axis of $y$; otherwise shearing strains are negative. A positive shearing strain is represented by $2 R T$ when $R T$ lies above the axis of $x$, and a negative shearing strain when $R T$ lies below the axis of $x$. For $-\frac{\pi}{2} \leqq \psi \leqq \frac{\pi}{2}$ any other shearing strain such as $2 R_{1} T$ is of the same sign as the strain $2 R T$ so long as $R_{1}$ lies on the same side of the diameter through $T$ as $R$. In figure $1(b)$, $2 R T$ represents a positive shearing strain and $2 R_{1} T$ a negative shearing strain.

Figure 2(a) shows three given strains, $\epsilon_{1}, \epsilon_{2}$, and $\epsilon_{3}$, of which $\epsilon_{1}$ and $\epsilon_{3}$ are negative and $\epsilon_{2}$ is positive, and figure $2(b)$ shows the construction of figure $1(b)$ applied to this case. In figure $2(b), \epsilon_{y}=R X, \gamma_{1}=$ $2 R_{1} T,-\epsilon_{x}=O R,-\gamma=2 R T$; that is, $\epsilon_{y}$ and $\gamma_{1}$ are positive, and $\epsilon_{x}$ and $\gamma$ are negative.

Usually the maximum and minimum strains-that is, the principal strains-and the numerically greatest shearing strain at a point are the strains it is particularly desired to know. Since strains are given as the intercepts obtained by dropping a perpendicular from $T$ on a diameter, it is clear that the maximum and minimum strains are obtrined when the diameter is intercepted by the point $T$ itself. Furthermore, since shearing strains are given as twice the perpendicular distance from ' $T$ ' to a diameter, it is clear that the numerically createst shenring strain is obtained when the diameter is perpendicular to the line connecting $T$ and the center of the circle. ${ }^{6}$ Thus in figures 3 and 4 , which are drawn for the same strains as figures 1 and 2, respectively, the principal strains are $\epsilon_{u}=V T, \epsilon_{v}=U T$ in directions making angles $\psi_{u}$ and $\psi_{0}\left(=\psi_{u}+\frac{\pi}{2}\right)$, respectively, with the axis of $x$. In figure 3 both $\epsilon_{u}$ and $\epsilon_{v}$ are positive. In figure $4 \epsilon_{u}$ is negative, and $\epsilon_{\mathrm{r}}$ is positive. The numerically greatest shearing strain in each of these figures is $\gamma_{\max }=2, Y T$ and occurs in the directions $x_{s}, y_{s}$.

- It twulif lo remembered that only conditions in one plane are heing considered here, and that in particular

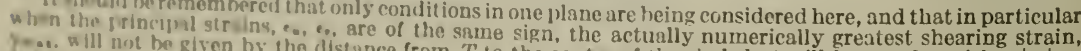

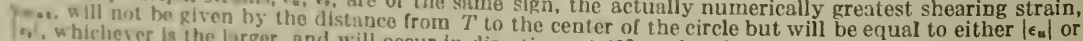
$\because$, whelies in the lirger, and will occur in directions at $45^{\circ}$ to the plane being considered. 
In practice it is convenient to take the direction of one of the given gage lines as that of the axis of $x$.

Strains on more than three gage lines.-If strains are measured on more than three gage lines through a point, the method just discussed may still be used; but now the circle will be overdetermined, since it

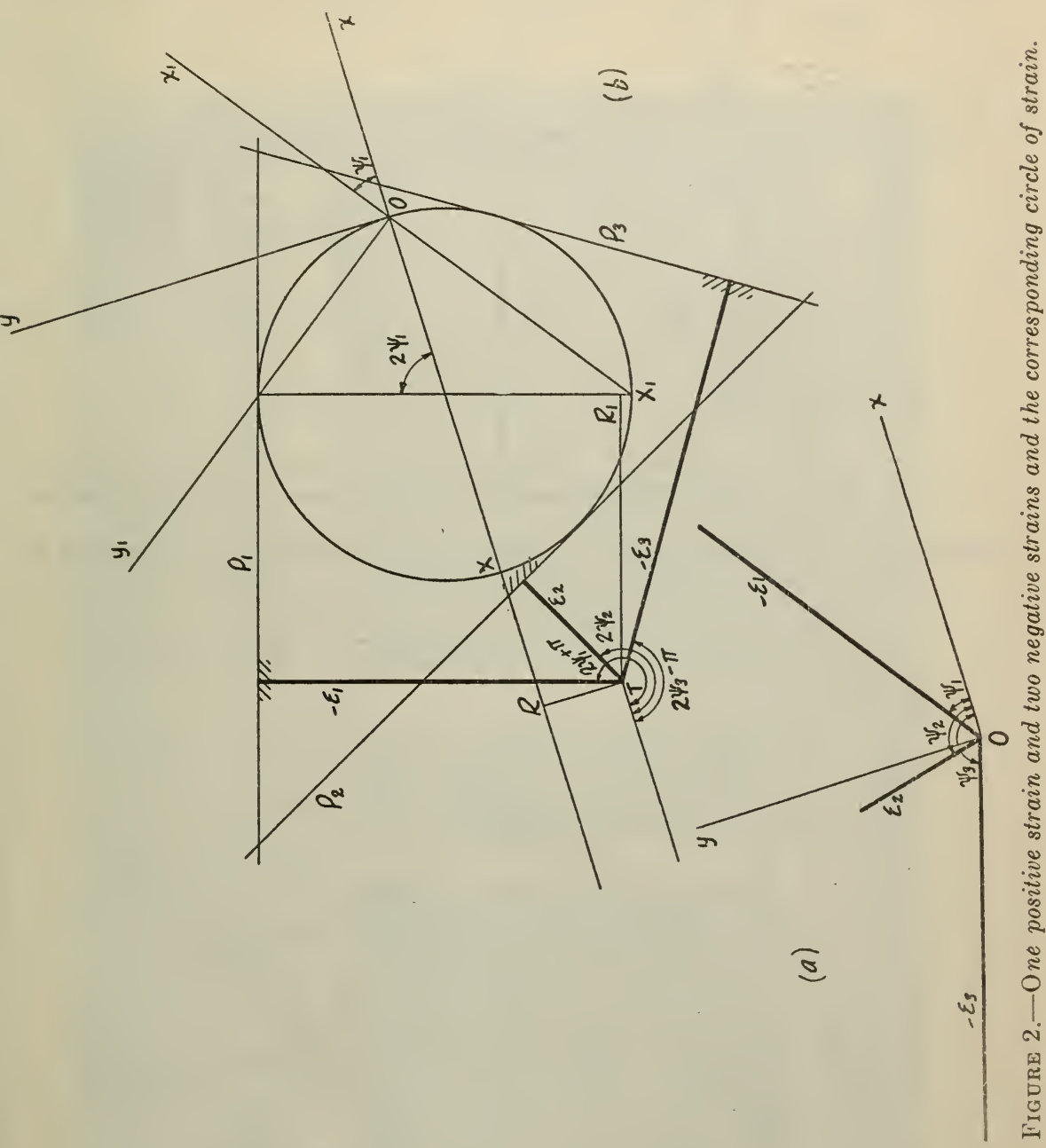

must be tangent to more than three lines. The procedure is to construct, as before, the perpendiculars $P_{1}, P_{2}, \ldots P_{n}$, where $n$ is the number of gage lines on which readings are taken, and then draw the circle in such a way as to make it as nearly as possible tangent to all the lines $P_{1}, P_{2}, \ldots P_{n}$.

When the principal strains and the numerically greatest shearing strain have been found, the principal stresses and the greatest shearing 


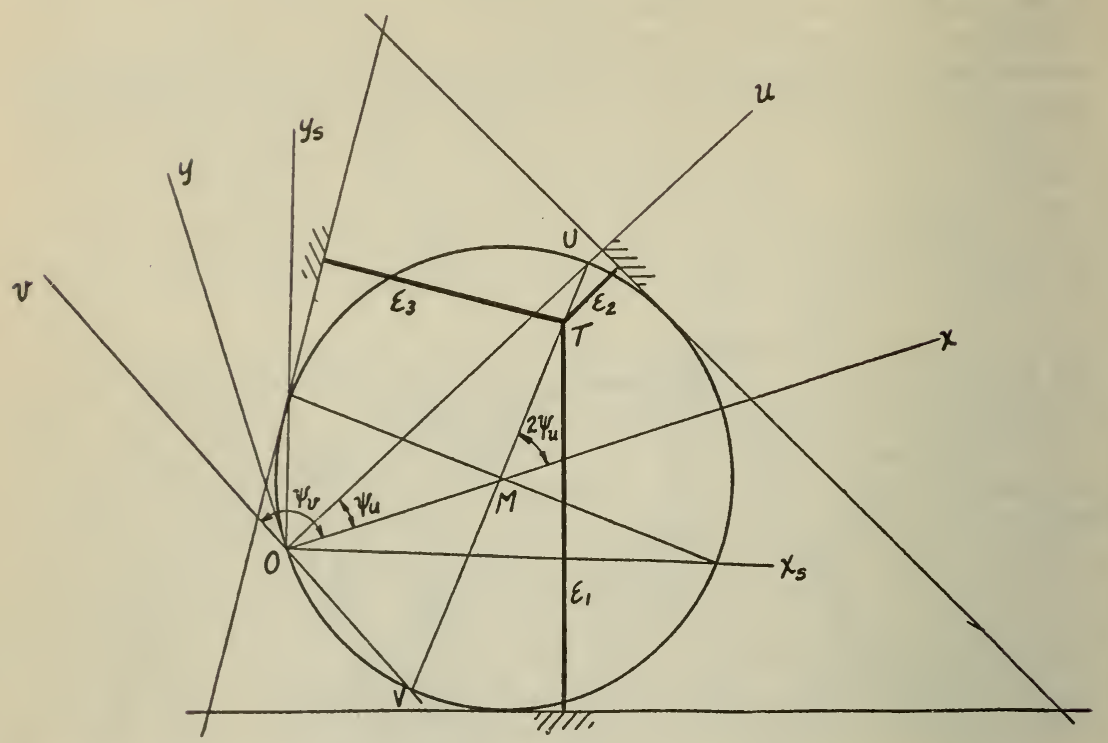

FIGURE 3.-Principal strains and maximum shearing strain for the case of figure 1.

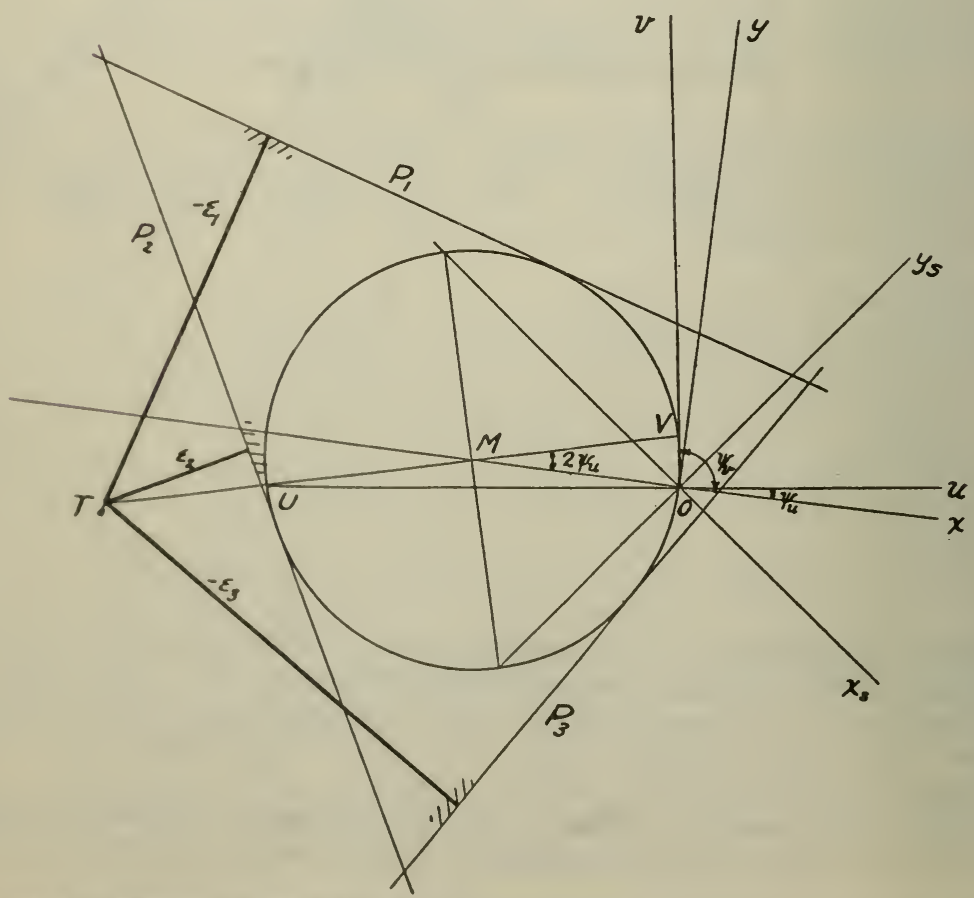

FIGUR: 4.-Principal strains and maximum shearing strain for the case of figure 2. 


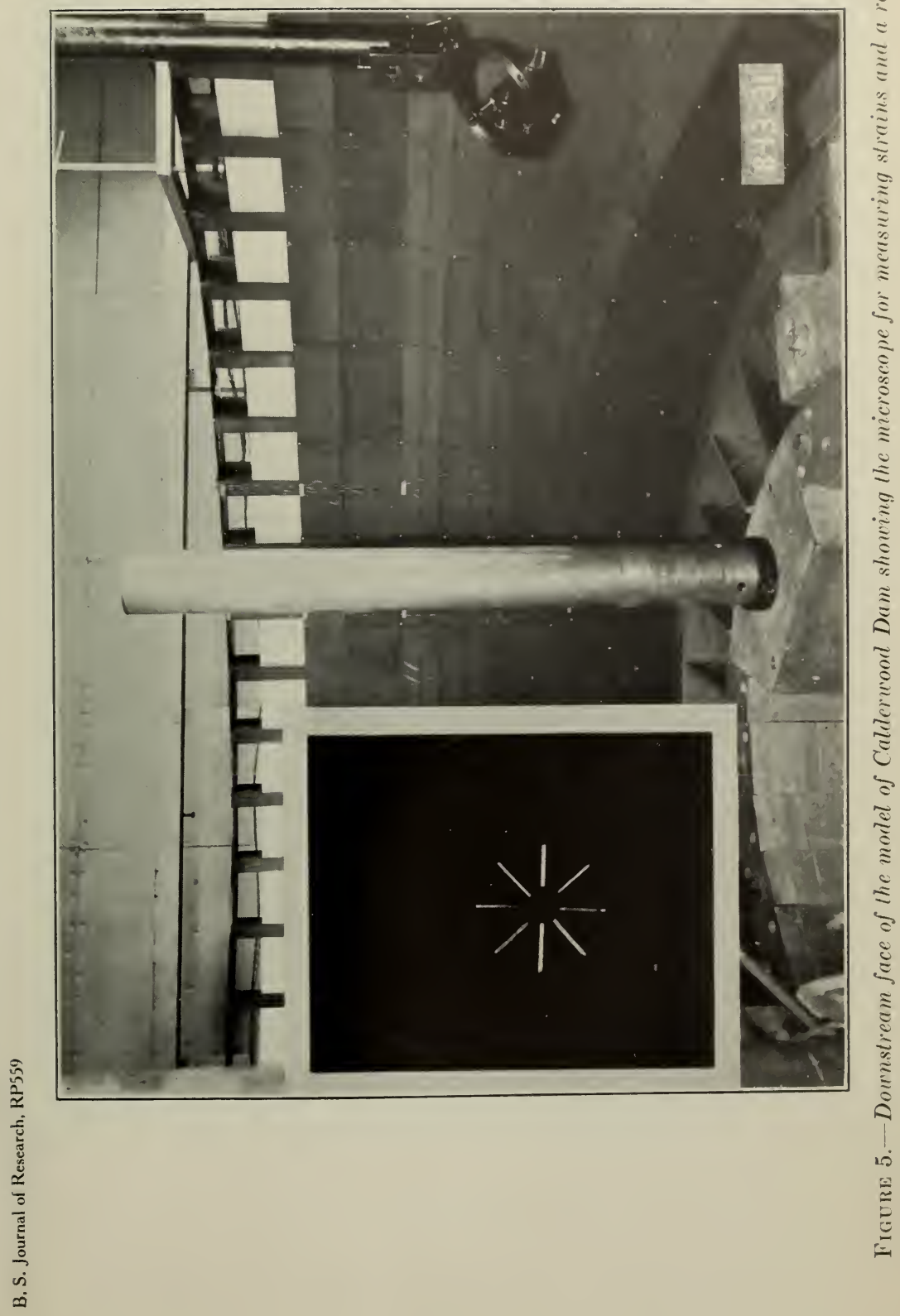




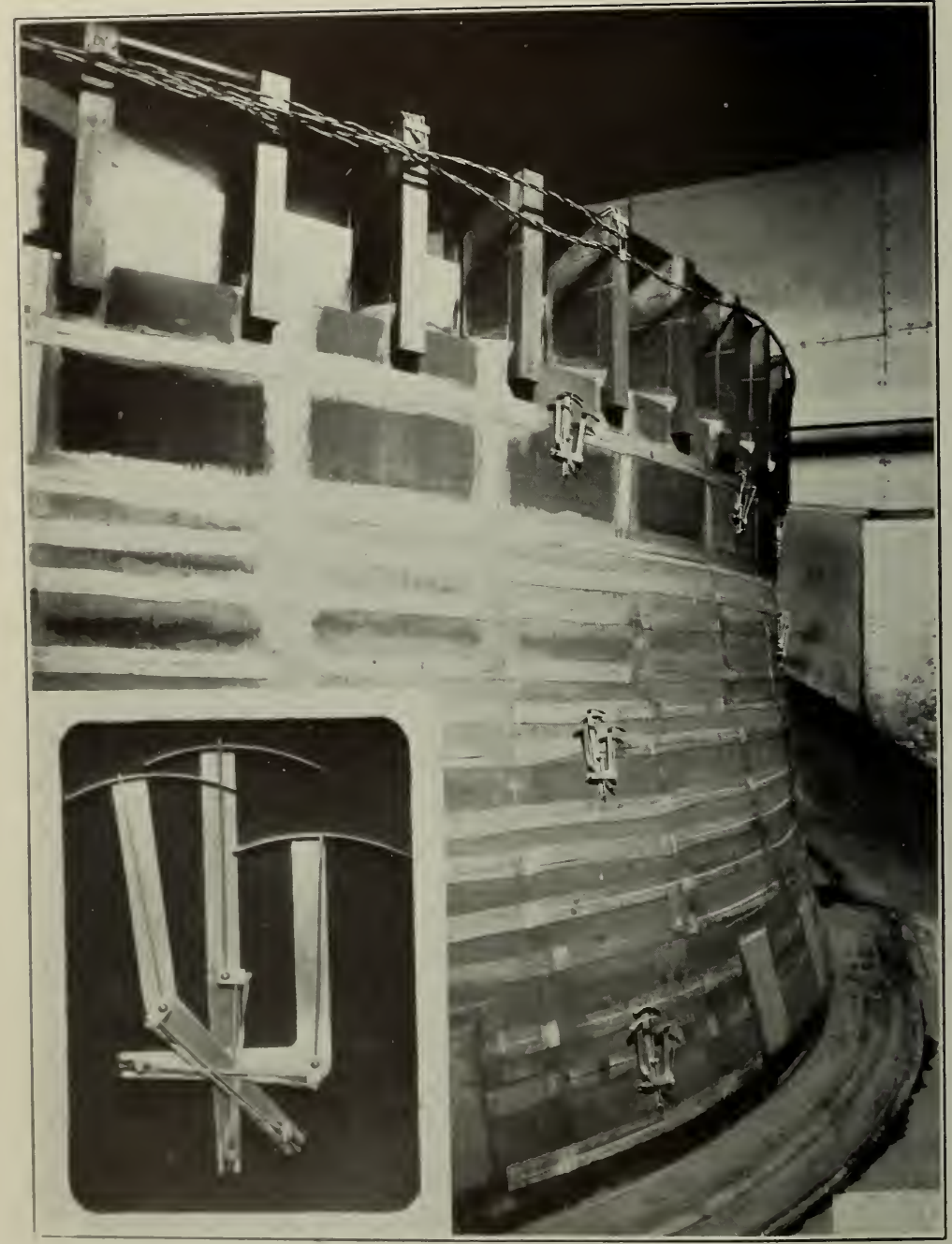

Fu, wr. b.-C'pstream face of the model of Calderwood Dam showing locations of roselles of three gage lines and the strain-measuring derice used, in insert. 


\section{B. S. Journal of Research, RP559}

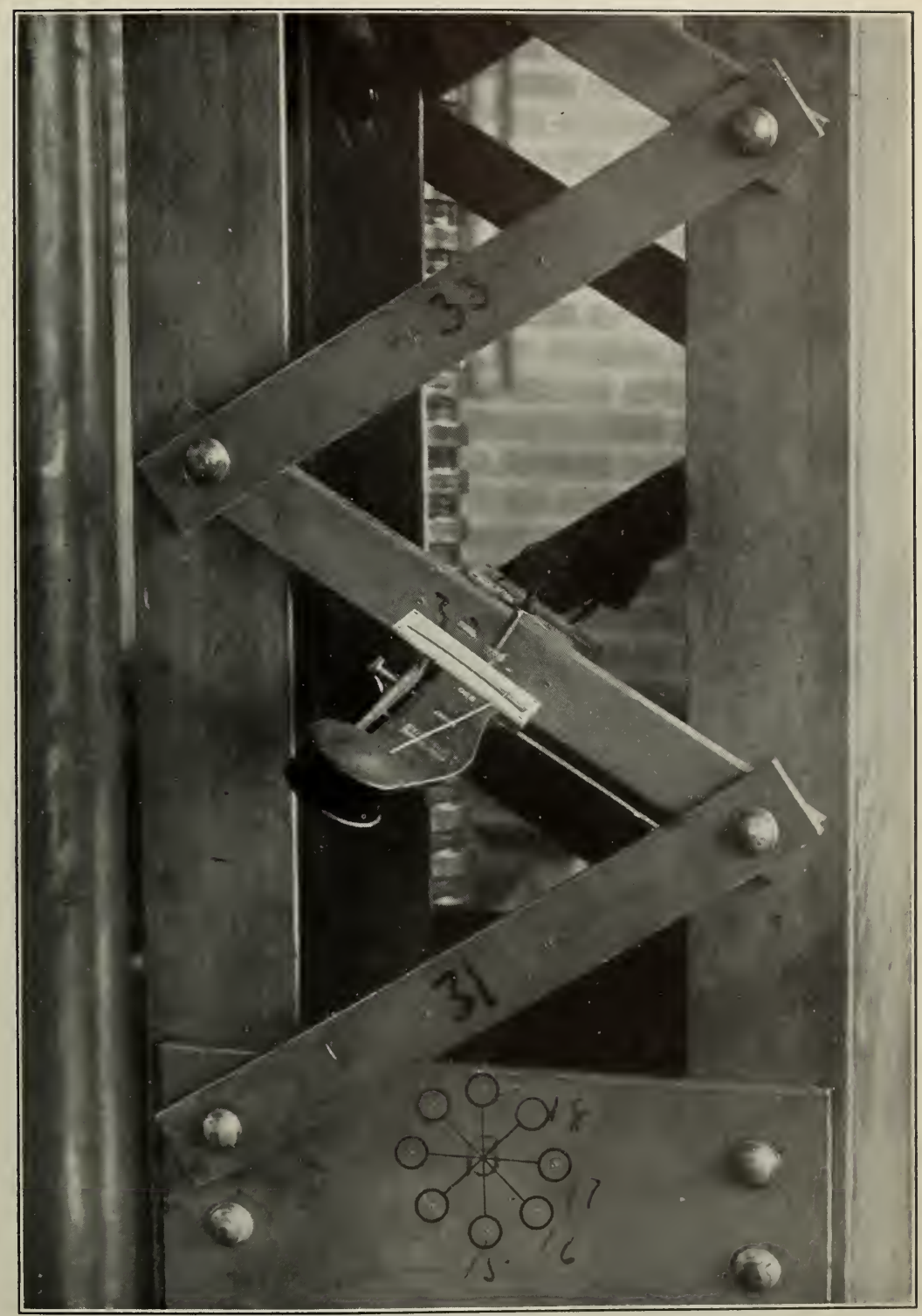

FIGURE 7.-Built-up aluminum alloy column showing a typical rosette in the end batten plate. 
B. S. Journal of Research, RP559

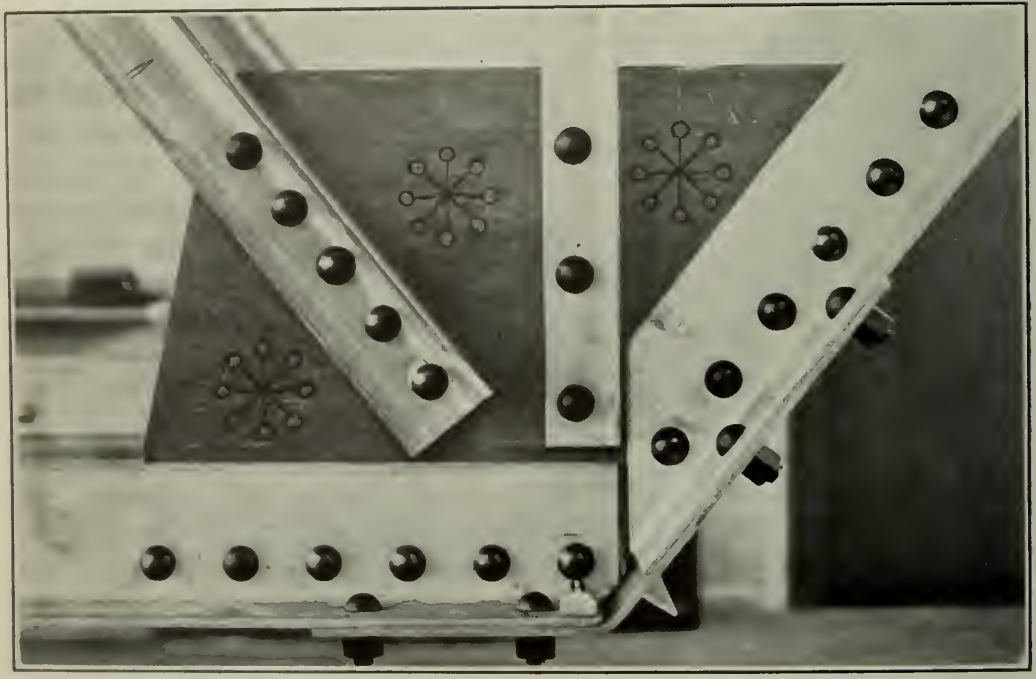

Figur: 9.- Giusset plate in all-aluminum truss showing rosettes of four 2-inch gage lines. 


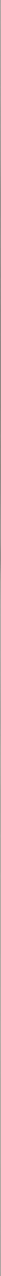


stress may be determined in an elastic body from the well-known relations

$$
\begin{gathered}
\sigma_{u}=\frac{m^{2} E}{m^{2}-1}\left(\epsilon_{u}+\frac{1}{m} \epsilon_{0}\right) \\
\sigma_{v}=\frac{m^{2} E}{m^{2}-1}\left(\epsilon_{v}+\frac{1}{m} \epsilon_{u}\right) \\
\tau_{\max }=G \gamma_{\max },\left(G=\frac{m E}{2(m+1)}\right)
\end{gathered}
$$

where

$\sigma_{u}$ and $\sigma_{o}$ are the principal stresses in the directions $u$ and $v$, respectively,

$\tau_{\max }$ is the greatest shearing stress,

$\frac{1}{m}$ is Poisson's ratio,

and

$E$ and $G$ are the modulus of elasticity and the shearing modulus of elasticity of the material, respectively.

\section{APPLICATION TO ACTUAL TESTS}

The graphical method just described has been applied to numerous cases of measurements of strain in structures and structural elements, such as dams, bearing plates, beams, and gusset plates, which have been tested in the past three or four years by the Aluminum Research Laboratories.

Tests of Calderwood Dam involved measurements of strain at 42 stations on the downstream face and at three stations on the upstream face. Each station on the downstream face consisted of four intersecting 10 -inch gage lines $45^{\circ}$ apart. The particular advantage of obtaining measurements of strain on sets of intersecting gage lines, or rosettes, on such a structure is the fact that the magnitude and the direction of the principal stresses may be found, thereby indicating the general direction which the thrust on the surface of the dam takes in reaching the foundation and abutments of the dam. Besides the principal stress the horizontal, vertical, or any inclined tensions and compressions as well as the shears in the surface of the dam are given by the strains on three or more gage lines. This information is extremely valuable in studying models of dams made of an elastic material. Strain measurements on four gage lines which are not consistent among themselves may be eliminated from the first determination of the general trend, and later the erroneous reading of the strain gage may readily be detected from this general trend. In this way the remaining three strains may be used to determine the dyadic circle for that point.

Strains were measured on rosettes of three and four gage lines on both the upstream and downstream face of a rubber-compound model of Calderwood Dam ${ }^{7}$ built and tested at the Aluminum Research Laboratories at New Kensington, Pa. Figure 5 is a general view of the downstream face of the model showing in the insert one of the

${ }^{7}$ A. V. Karpov and R. L. Templin, Building and Testing an Arch Dam Model, Civil Engineering, January 1932. 
four gage-line rosettes which were used. Changes in gage length were measured with the filar microscope shown in the photograph. Figure 6 shows the type of three gage-line rosette used on the upstream face of the model. With strains measured on these rosettes dyadic circles were constructed from which the principal stresses and maximum shears were obtained.

The stresses in the webs of I-beams carrying concentrated loads in the case of either long, thin-webbed beams or heavier beams on short spans may be complicated by local stresses as well as by the primary shearing stresses. Measurements of strain on rosettes of three and four gage lines on the webs of aluminum-alloy I-beams and plate girders under concentrated loads have yielded direct information concerning these stresses by means of the application of the principles heretofore described.

The use of measurements of strain on rosettes for the determination of the shear in end batten plates of built-up columns under eccentric and oblique loads has proved to be very satisfactory. Figure 7 shows a typical rosette on a built-up aluminum-alloy column ready to be tested. From the results of these measurements of strain the general character of the distribution of shear in the plate was determined. Figure 8 shows the measured strains, the corresponding dyadic circles, and the principal stresses obtained for the particular cases of loading indicated.

One of the most interesting applications of measurements of strain on rosettes is that of finding the stresses in gusset plates where several members are riveted to a single plate. Figure 9 shows rosettes locrted on a gusset in an aluminum-alloy truss, tested to determine the transmission of stresses through gusset plates and riveted connections.

An instance of the employment of rosettes of short gage lengths (one-half inch) may be cited to demonstrate another field of application of these principles. The stresses occurring in the front end of an aluminum-alloy locomotive side rod were determined both in magnitude and direction by the use of rosettes strategically placed on the side near the end of the rod. The use of four gage lines shows the limits of accuracy of measurement by the differences in the radii of the four dyadic circles drawn from the various combinations of strains on any three of the four lines.

The examples given illustrate the wide range of cases to which such measurements of strain may be applied. It is only through some such measurements that a complete picture of the state of stress in a body of two principal dimensions or in the surface of a body of three dimensions con be obtained.

Wasington, February 14, 1933. 